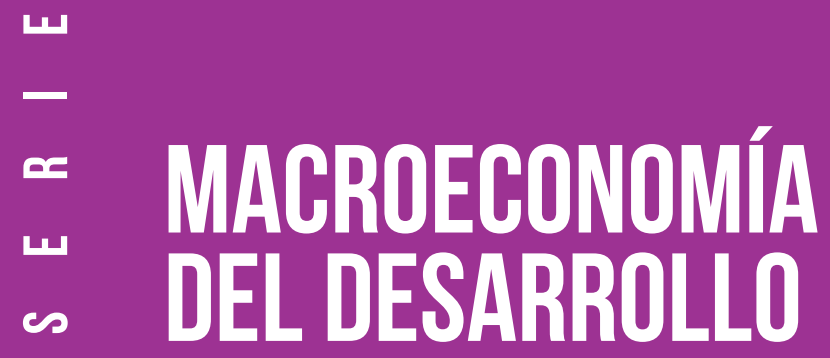

El desempeño mediocre de la productividad laboral en América Latina: una interpretación neoclásica

Claudio Aravena

Juan Alberto Fuentes 


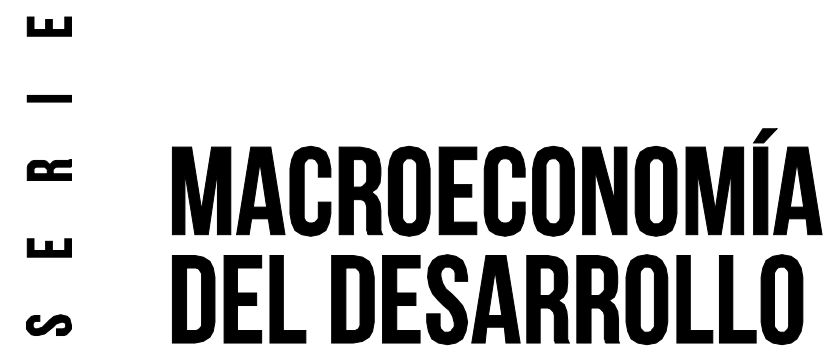

140

\section{El desempeño mediocre de la productividad laboral en América Latina: una interpretación neoclásica}

Claudio Aravena

Juan Alberto Fuentes

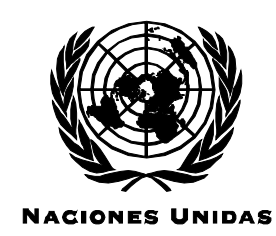

C E P \& L 
Este documento fue preparado por Claudio Aravena y Juan Alberto Fuentes, de la División de Desarrollo Económico de la Comisión Económica para América Latina y el Caribe (CEPAL). Se agradece a André Hofman, director del proyecto CEPAL-LA KLEMS, por los datos de Argentina, Brasil, Chile y México, Carolina Cavada por su cooperación en la elaboración de las series de pago de los factores con redistribución del ingreso mixto y los valiosos comentarios de Matilde Mas y de los asistentes al taller interno de la División de Desarrollo Económico de CEPAL, Naciones Unidas, agosto 2013.

Las opiniones expresadas en este documento, que no ha sido sometido a revisión editorial, son de exclusiva responsabilidad de los autores y pueden no coincidir con las de la organización.

Publicación de las Naciones Unidas

ISSN 1680-8843

LC/L.3725

Copyright $($ N Naciones Unidas, noviembre de 2013. Todos los derechos reservados

Impreso en Naciones Unidas, Santiago de Chile

Los Estados miembros y sus instituciones gubernamentales pueden reproducir esta obra sin autorización previa. Solo se les solicita que mencionen la fuente e informen a las Naciones Unidas de tal reproducción. 


\section{Índice}

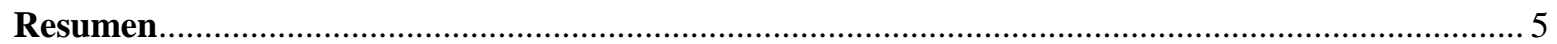

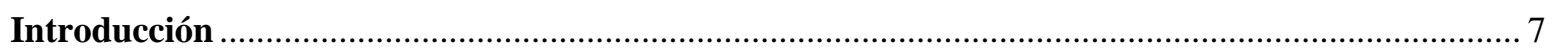

I. Evolución de la productividad laboral en América Latina entre 1981 y 2010 ......................... 9

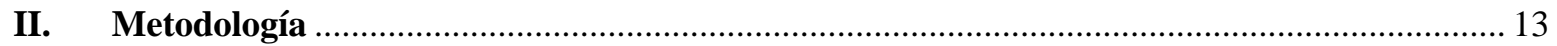

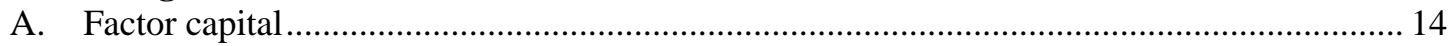

1. Acervo de capital ....................................................................................................... 14

2. Costo de uso del capital ................................................................................................ 15

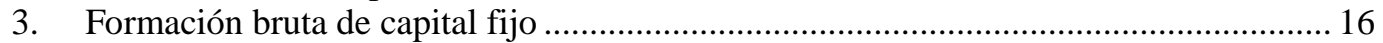

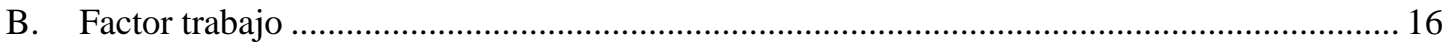

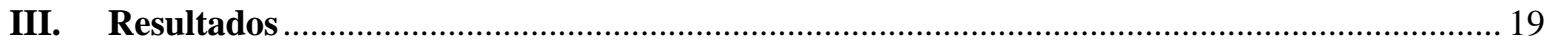

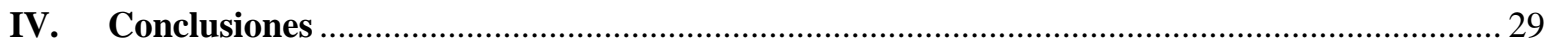

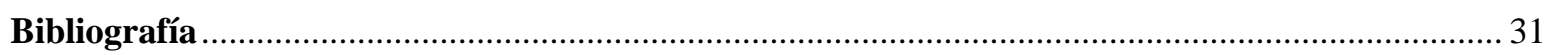

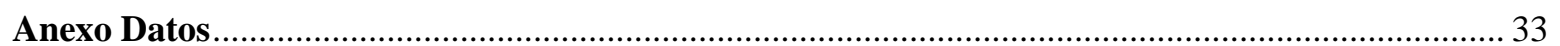

Serie Macroeconomía del Desarrollo: números publicados............................................................. 36

Índice de cuadros

CUADRO 1 AMÉRICA LATINA: PRODUCTO INTERNO BRUTO POR HORA TRABAJADA COMO PORCENTAJE DE ESTADOS UNIDOS, 1980-2010 …..................................12

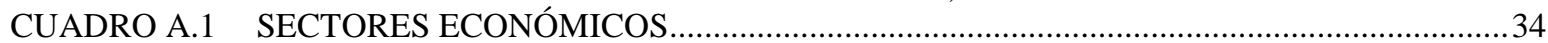

CUADRO A.2 DESAGREGACIÓN DE LA FBCF POR TIPO DE ACTIVO ............................................34

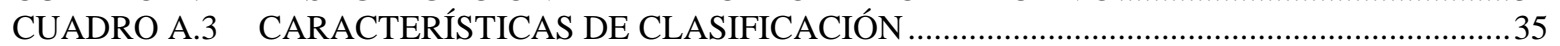

Índice de gráficos

GRÁFICO 1 AMÉRICA LATINA: TASA DE VARIACIÓN DEL VALOR AGREGADO, HORAS TRABAJADAS Y PRODUCTIVIDAD LABORAL ..........................................10

GRÁFICO 2 AMÉRICA LATINA: TASA DE VARIACIÓN DEL VALOR AGREGADO, HORAS TRABAJADAS Y PRODUCTIVIDAD LABORAL, 1981-2010 …………….........10

GRÁFICO 3 AMÉRICA LATINA: EVOLUCIÓN DE LA PRODUCTIVIDAD LABORAL, $1980-2010$ 
GRÁFICO 4 AMÉRICA LATINA: CONTRIBUCIONES DE LA RELACIÓN CAPITAL POR HORA AL CRECIMIENTO DE LA PRODUCTIVIDAD LABORAL, 1981-2010

GRÁFICO 5 AMÉRICA LATINA: DETERMINANTES DE LA PRODUCTIVIDAD LABORAL, MÉTODO TRADICIONAL Y NO TRADICIONAL, 1981-2010.

GRÁFICO 6 AMÉRICA LATINA: DETERMINANTES DE LA PRODUCTIVIDAD

LABORAL, 1980-2010.

GRÁFICO 7 ARGENTINA, BRASIL, CHILE Y MÉXICO: DETERMINANTES DE LA PRODUCTIVIDAD LABORAL, 1994-2008..

GRÁFICO 8 ARGENTINA, BRASIL, CHILE Y MÉXICO: DETERMINANTES

GRÁFICO 9 DE LA PRODUCTIVIDAD LABORAL POR AÑO, 1994-2008 24 ARGENTINA, BRASIL, CHILE Y MÉXICO: DETERMINANTES

DE LA PRODUCTIVIDAD LABORAL POR SECTOR, 1994-2008. 26 


\section{Resumen}

Este documento examina las experiencias de crecimiento de la productividad laboral de Argentina, Bolivia (Estado Plurinacional de), Brasil, Chile, Colombia, Costa Rica, Ecuador, Guatemala, Honduras, México, Nicaragua, Panamá, Paraguay, Perú, Uruguay y Venezuela (República Bolivariana de). El análisis arroja luz sobre los puntos fuertes y débiles de crecimiento a largo plazo de estos países mediante la identificación de similitudes y diferencias con otros países y evalúa su desempeño económico en esa base de comparación.

En orden a analizar cuan satisfactorio ha sido el desempeño de la productividad laboral en los últimos 30 años nos centramos en su tasa de crecimiento y de los factores que contribuyen a ella.

En el presente trabajo se realiza una estimación de los factores que contribuyen a la productividad laboral para el periodo 1980-2010, medido a través de la aplicación de la metodología de la contabilización del crecimiento, pero a diferencia de los estudios tradicionales los esfuerzos se concentraron en cuantificar el aporte de las diferentes características del empleo y en una mayor desagregación de los activos del capital, incorporando de manera especial las tecnologías de la información y comunicación, que cobran relevancia desde la década de los 90's, cuyos resultados muestran diferencias en el aporte del capital al crecimiento calculado a través de la agregación de sus activos utilizando su costo de uso y no su valor de mercado como ponderador. Para este análisis, estudiamos los dieciséis países antes mencionados, desagregando el capital en cuatro tipos de activos y distinguiendo el nivel de estudio del factor trabajo. 



\section{Introducción}

En este trabajo se realiza una estimación de los factores que contribuyeron a la productividad laboral en América Latina ${ }^{1}$ para el periodo 1980-2010, medido a través de la aplicación de la metodología de la contabilización del crecimiento. A diferencia de los estudios tradicionales, los esfuerzos se concentraron en cuantificar el aporte de las diferentes características del empleo, distinguiendo el nivel de estudio del factor trabajo, y se desagregan los activos del capital. En particular, se estima para 16 países el aporte de las tecnologías de la información y comunicación incorporadas a la inversión, mediante la desagregación del capital en cuatro tipos de activos, analizados con base en su costo de uso y no su valor de mercado como ponderador ${ }^{2}$.

De manera complementaria se utiliza la base de datos LAKLEMS ${ }^{3}$ para desagregar en nueve sectores económicos, y en cada uno de ellos se distingue tres características del factor trabajo (sexo, edad y nivel de estudio) y ocho tipos de activos referidos al capital. Estos datos son menos extensos, pues cubren el período 1994-2008, y tienen una cobertura de países limitada, disponible solo para Argentina, Brasil, Chile y México.

El trabajo está organizado de la siguiente forma. En la primera sección se presenta una visión general sobre la evolución de la productividad laboral en América Latina durante las últimas tres décadas. El desempeño poco satisfactorio de la productividad en la región que surge de este panorama es la principal motivación de este estudio, que busca dar respuesta sobre cuáles son sus principales determinantes y la forma correcta de medirlos.

1 En un primer análisis global se evalúa el desempeño de 16 países: Argentina, Bolivia (Estado Plurinacional de), Brasil, Chile, Colombia, Costa Rica, Ecuador, Guatemala, Honduras, México, Nicaragua, Panamá, Paraguay, Perú, Uruguay y Venezuela (República Bolivariana de). Luego se realiza un análisis sectorial restringido a 4 países: Argentina, Brasil, Chile y México.

2 Véase Vries de, G., Mulder, N., Borgo dal, M., and Hofman, A. (2007) para un estudio reciente donde se desagrega la inversión en tecnologías de información y comunicaciones.

3 El proyecto LA-KLEMS es coordinado por la Comisión Económica para América Latina y el Caribe (CEPAL) de las Naciones Unidas. El objetivo de esta iniciativa es la construcción de estadísticas de productividad comparables a nivel de sectores para países de América Latina. Las siglas provienen del uso de una función de producción que contabiliza, además del capital (K) y el trabajo (L), insumos de energía (E), materiales (M) y servicios (S). Para mayores detalles, véase Hofman y Aravena (2013) y www.cepal.org/la-klems. 
La sección dos describe la metodología neoclásica de cálculo de los determinantes del crecimiento y el cálculo de los factores de producción. La sección tres presenta los resultados y se examinan las fuentes del crecimiento, tanto para los cálculos agregados de los dieciséis países como para los sectoriales de los cuatro países de análisis. Por último, se presentan las conclusiones. 


\section{Evolución de la productividad laboral en América Latina entre 1981 y 2010}

$\mathrm{Al}$ descomponer el crecimiento del valor agregado en el cambio en las horas trabajadas y en el cambio de la productividad (valor agregado por hora), se puede observar que el crecimiento promedio de 2,8\% del valor agregado entre 1981 y 2010 para el conjunto de los países analizados se explica por el aumento de las horas trabajadas, mientras que la productividad laboral se redujo en -0,3\% (véase el gráfico 1 ). Ello esconde diferencias entre países, así como tendencias cambiantes. Por una parte, en la mitad de los países el aumento de horas trabajadas fue complementado por un débil aumento de la productividad laboral, a excepción de Argentina y Chile, donde el aumento de la productividad explica la mitad del incremento del valor agregado. En el resto de los países, ocho de dieciséis, la productividad laboral disminuyó, lo cual fue compensado por un aumento de los horas trabajadas, que fue mayor al incremento del valor agregado (véanse los gráficos 1 y 2).

Por otra parte, los resultados por décadas muestran que en la década de los ochenta el valor agregado promedio aumentó en sólo 1,3\% y únicamente dos países, Colombia y Honduras, experimentaron aumentos de su productividad laboral. Durante la década de los noventa hubo una mejora tanto en el valor agregado como en la productividad laboral, y siete países registraron aumentos de su productividad laboral, destacándose Argentina y Chile, donde este aumento explica en torno a tres cuartos del crecimiento del valor agregado y Uruguay, donde lo explica completamente. En el periodo 2001-2010, cuando el valor agregado creció 3,8\%, trece de los 16 países tuvieron aumentos de la productividad laboral, aunque sin recuperar los niveles de productividad que existían a principios de la década de 1980 en varios casos. Entre los que más aumentaron su productividad está claramente Chile (gráfico 3, primer panel), mientras que ocho países terminaron con una productividad laboral menor a la observada treinta años antes, con un declive fuerte en la primera década que no fue compensado a pesar de aumentos de la productividad en años posteriores (véase el gráfico 3, segundo panel). 


\section{GRÁFICO 1 \\ AMÉRICA LATINA: TASA DE VARIACIÓN DEL VALOR AGREGADO, HORAS TRABAJADAS Y PRODUCTIVIDAD LABORAL}

(Promedio de 16 países)

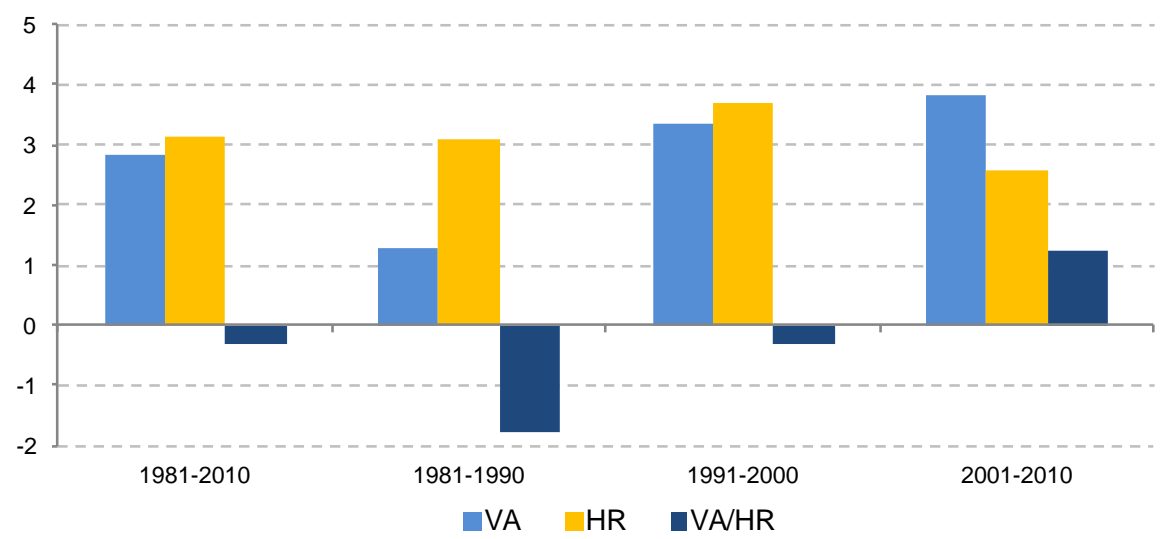

Fuente: Elaboración propia sobre la base de datos oficiales de los países y OIT.

GRÁFICO 2

AMÉRICA LATINA: TASA DE VARIACIÓN DEL VALOR AGREGADO, HORAS TRABAJADAS Y PRODUCTIVIDAD LABORAL, 1981-2010

1981-2010
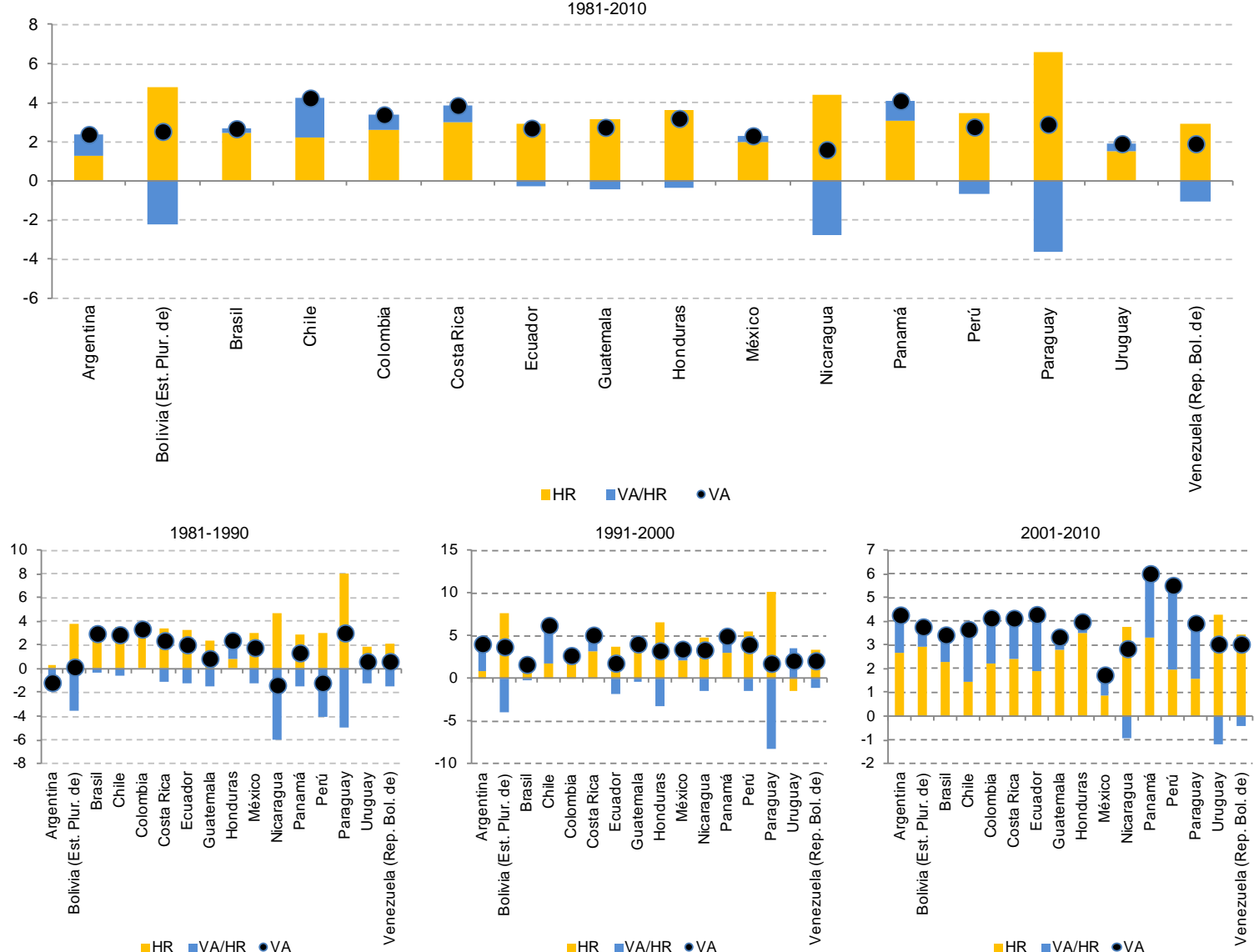

Fuente: Elaboración propia sobre la base de datos oficiales de los países y OIT. 


\section{GRÁFICO 3 \\ AMÉRICA LATINA: EVOLUCIÓN DE LA PRODUCTIVIDAD LABORAL, 1980-2010}

$(1980=100)$
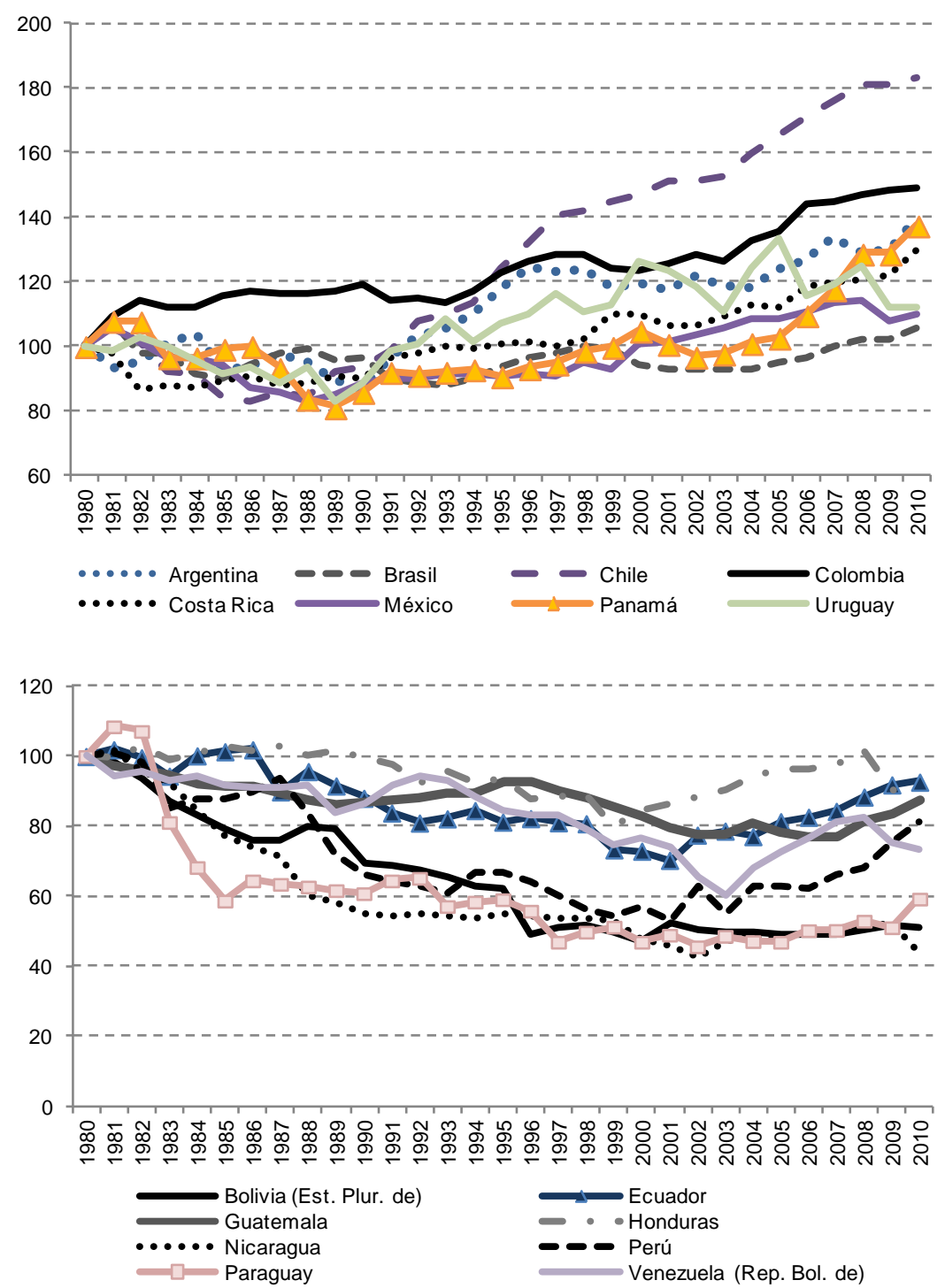

Fuente: Elaboración propia sobre la base de datos oficiales de los países y OIT.

La comparación de la productividad de los países de la región con la de los Estados Unidos muestra que la brecha entre el conjunto de países y este país aumentó, con la única excepción de Chile (véase el cuadro 1). Chile logró recuperar el punto de partida de inicios de los ochenta respecto a Estados Unidos para luego superarlo a finales de los noventa y consolidar este avance durante la década siguiente. 


\section{CUADRO 1}

AMÉRICA LATINA: PRODUCTO INTERNO BRUTO POR HORA TRABAJADA COMO PORCENTAJE DE ESTADOS UNIDOS, 1980-2010

(En porcentajes)

\begin{tabular}{lrrrr}
\hline & 1980 & 1990 & 2000 & 2010 \\
\hline Argentina & 36 & 28 & 31 & 27 \\
Bolivia (Estado Plurinacional de) & 21 & 13 & 07 & 07 \\
Brasil & 30 & 22 & 19 & 18 \\
Chile & 37 & 30 & 39 & 41 \\
Colombia & 23 & 21 & 17 & 17 \\
Costa Rica & 26 & 21 & 21 & 21 \\
Ecuador & 29 & 22 & 15 & 16 \\
Guatemala & 23 & 17 & 13 & 12 \\
México & 38 & 29 & 27 & 26 \\
Perú & 40 & 23 & 16 & 19 \\
Uruguay & 40 & 30 & 38 & 28 \\
Venezuela (República Bolivariana de) & 61 & 46 & 34 & 27 \\
\hline
\end{tabular}

Fuente: Elaboración propia sobre la base de datos oficiales de los países, OIT y Conference Board. 


\section{Metodología}

El marco teórico utilizado para analizar la evolución de la productividad en este caso es el enfoque neoclásico tradicional, centrado en dos fuentes del crecimiento económico, capital y trabajo, y para medir su contribución al crecimiento económico la metodología utilizada es la conocida como contabilidad del crecimiento. Su origen se encuentra en la obra de Jorgenson y Griliches (1967) ${ }^{4}$.

La metodología empleada se basa en la frontera de posibilidades de producciones (FPP), que describe combinaciones eficientes de producción e insumos para la economía en su conjunto, y toma la forma siguiente:

$$
Y=A \cdot f(K, L)
$$

donde $Y$ es el producto agregado, $K$ y $L$ son el capital y el trabajo, y A es un factor, neutral en el sentido de Hicks, que aumenta el volumen agregado de insumos. Como aporte de este estudio el marco analítico —y la medición correspondiente— se amplía para destacar la composición de los insumos del capital y del trabajo como:

$$
Y=A \cdot f\left(K\left(K_{\text {tic }}, K_{\text {notic }}\right), L\left(H, L_{Q}\right)\right)
$$

donde $K_{\text {tic }}$ y Knotic son el capital de activos TIC y activos no TIC, respectivamente. H es el total de horas trabajadas y LQ es la calidad del trabajo. Es necesaria la estimación de la contribución del capital TIC por la oleada de inversiones TIC causadas por la brusca aceleración en el ritmo de descenso de los precios de del hardware y software, que comenzó en 1995. Ello es congruente con la observación de Abramovitch (1994), de ajustar las estimaciones del crecimiento y de sus fuentes tomando en cuenta las características más específicas del capital en cada momento histórico. Estas características del capital también contribuyen a explicar lo que de otra manera pareciera ser explicado por la Productividad Total de Factores, factor residual que corresponde a A en las siguientes ecuaciones.

\footnotetext{
$4 \quad$ Y luego en Jorgenson, Gollop y Fraumeini (1987) y Jorgenson, Ho y Stiroh (2005).
} 
Suponiendo que los mercados de productos y factores son competitivos, el marco ampliado implica la siguiente descomposición:

$$
\Delta \ln Y=V_{\text {Ktic }} \Delta \ln K_{\text {tic }}+V_{\text {Knotic }} \Delta \ln K_{\text {notic }}+V_{L} \Delta \ln H+V_{L} \Delta \ln L_{Q}+\Delta \ln A
$$

Cada $v$ representa la participación en los ingresos totales del insumo expresado en el subíndice. Es decir, el crecimiento de la producción puede ser descompuesto en contribuciones del capital TIC, capital no TIC, horas de trabajo, calidad del trabajo y un factor residual (A), que la literatura neoclásica generalmente califica como productividad total de los factores. La ecuación (3) puede también plantearse en términos de los determinantes de la productividad laboral, cuyos factores son la relación capital por hora, la calidad del trabajo y un residual:

$$
\Delta \ln Y / H=V_{\text {Ktic }} \Delta \ln K_{\text {tic }} / L_{L}+V_{\text {Knotic }} \Delta \ln K_{\text {notic }} / L+\Delta \ln L_{Q}+\Delta \ln A
$$

Existen varias fuentes de error en la medición de las variables de la ecuación (3), pero son relativamente menores en comparación con el problema que presenta por un lado la definición y medición de acervos de capital y sus servicios y por otro los servicios relativos del empleo (Jorgenson and Griliches, 1967) ${ }^{5}$.

\section{A. Factor capital}

De manera similar a que los trabajadores son repositorios del acervo de capital humano y prestan servicios que pueden ser medidos en horas trabajadas, los bienes de capital representan un acervo que provee un flujo de servicios ${ }^{6}$, que constituyen insumos en el proceso productivo. La diferencia es que mientras los trabajadores reciben una remuneración a cambio de los servicios prestados, en general, los bienes de capital son propiedad de la empresa que los utiliza, por lo que no existe un registro de la remuneración de los servicios prestados por los bienes de capital.

En consecuencia, la medición del aporte del factor capital en el proceso productivo plantea la correcta estimación de los flujos de los servicios derivados de los distintos tipos de activos de capital, así como de los ponderadores utilizados en su agregación. La estimación de los flujos de servicios de capital se inicia a través de la medición de los acervos de capital disponibles en el tiempo. Una vez que el acervo de capital ha sido estimado, se calcula su respectivo costo de uso, el cual es utilizado para agregar los distintos tipos de activos en un índice de servicios de capital.

\section{Acervo de capital}

El stock de capital neto es definido como la suma ponderada de las inversiones pasadas de los diferentes tipos de activos productivos disponibles en la economía. La ponderación esta definida por la eficiencia relativa de los distintos activos de diferentes edades. El stock de capital neto para el activo j se estima a partir de la siguiente formula:

$$
K_{t, j}^{p}=\sum_{\tau=0}^{T_{j}} I_{j, t-\tau} R_{j, \tau} E_{j, \tau}
$$

donde $I_{j, t-\tau}$ es la inversión de edad $\tau$ expresada a precios constantes; $R_{j, \tau}$ es la función de retiro, la que determina la proporción de la inversión realizada en $\tau$ periodos y que sobrevive actualmente; y, $E_{j, \tau}$ representa el perfil edad-eficiencia, que caracteriza la pérdida de eficiencia productiva de los activos según envejecen. Se utiliza una depreciación geométrica para modelar tanto el retiro, como la pérdida de eficiencia., ambos componentes son reemplazados por la tasa de depreciación.

Griliches (1994) ha argumentado que la certeza con la cual se puede estimar el aporte del capital y del empleo está limitada por la disponibilidad de datos. Ver Griliches1994 para una discusión detallada.

6 El ejemplo clásico es el de una bodega que provee un flujo de servicios medido en volumen de almacenaje. 
Una vez que se ha estimado el stock de capital neto para cada tipo de activo, el siguiente paso es agregar los activos. El supuesto de competencia perfecta en el mercado de factores implica que una empresa maximizadora de beneficios utilizará bienes de capital hasta el punto en el que la renta pagada sea igual al beneficio marginal del bien. Por ello, la agregación de los servicios de capital de distintos tipos de activos se lleva a cabo utilizando como ponderador el costo de uso de capital.

\section{Costo de uso del capital}

En equilibrio, y dados los supuesto que se han hecho, el precio de mercado de un activo sería igual al valor presente esperado de los flujos generados por el mismo. En el caso de los bienes de capital los flujos son equivalentes a lo que su dueño recibiría por rentar el activo durante cierto periodo. Por ello, el valor de mercado de un activo con vida máxima $T_{j}$, de edad $\tau$ en el momento $t$ estaría dado por:

$$
p_{j, t, \tau}=\sum_{s=0}^{T_{j}}\left[\frac{\mu_{j, t+s, \tau+s}}{\prod_{k=0}^{s}\left(1+i_{t+k}\right)}\right]
$$

donde $i_{t}$ es la tasa nominal de retorno, la cual se supone que es igual para todos los tipos de activos; y $\mu_{j, t, \tau}$ es el monto recibido por rentar el activo de edad $\tau$ durante el periodo $t$, o costo de uso, el cual bajo los supuestos realizados es igual al producto marginal del activo y está expresado como:

$$
\mu_{j, t, 0} \approx p_{j, t, 0}\left(i_{t}+d_{j, t, 0}-q_{j, t}\right)
$$

En este documento el costo de uso se estima utilizando una tasa de retorno exógena, obtenida a partir de las tasas de interés observables en el mercado. El problema es que la tasa relevante depende del perfil de financiamiento de cada empresa, por lo que se suele utilizar un promedio de las tasas activas y pasivas.

La utilización de una tasa de retorno exógena supone implícitamente (Harchaoui Tarkhani, 2002) que los agentes económicos tienen información completa, lo cual implica que no existen problemas de agencia entre los dueños de los factores de producción y quienes los administran, a la vez que existe un mercado completo y eficiente de activos de segunda mano, lo cual implica que las decisiones de inversión son reversibles, que los activos de capital son divisibles y que los distintos tipos de activos son sustitutos en el proceso productivo.

Una de las consecuencias de adoptar una tasa de retorno exógena, es que en general el valor total de los servicios de capital no será igual al excedente bruto de explotación obtenido a partir de las cuentas nacionales. Esta discrepancia se puede explicar como una diferencia entre los costos esperados y los realizados, como evidencia de que el proceso productivo no exhibe rendimientos constantes a escala o a la existencia de mercados no competitivos.

En particular, se supone que la tasa de retorno nominal esta dada por la fórmula de Fisher: $1+i_{t}=(1+r)\left(1+\pi_{t}\right)$; donde tal como en Mas, M., Pérez, F., and Uriel, E. (2005) se supone que $r=4 \%$, lo cual es aproximadamente el promedio histórico de la tasa de interés libre de riesgo en la OCDE.

Una vez estimados los costos de uso de capital para cada tipo de activo, se puede obtener las variaciones del índice de valor de los servicios de capital utilizando un índice de Tornqvist (1936) ${ }^{7}$ :

$$
\Delta \zeta_{t, K}=\Pi_{j}\left(\frac{K_{j, t}^{p}}{K_{j, t-1}^{p}}\right)^{\bar{v} j}
$$

en donde los ponderadores se definen como:

$$
\bar{v}_{j}=0.5\left(v_{j, t}+v_{j, t-1}\right)
$$

$$
v_{j, t}=\frac{\mu_{j, t} K_{j, t}^{p}}{\sum_{j} \mu_{j, t} K_{j, t}^{p}}
$$




\section{Formación bruta de capital fijo}

La formación bruta de capital fijo (FBCF) es insumo indispensable para el cálculo del acervo neto de capital, ya que está compuesta de bienes utilizados para producir o generar valor en un proceso productivo, por lo que su desagregación por tipo de activo es de suma importancia para realizar la correcta estimación de los flujos de servicios de capital, como también para descomponer el aporte al crecimiento de cada activo. La diferenciación por tipo de activo permite distinguir el papel que juegan los activos TIC versus los no TIC en el crecimiento económico.

Dado que los activos TIC se han integrado fuertemente en las últimas décadas, y de forma dispar entre las economías en estudio, las cuentas nacionales hoy cuentan con series oficiales de inversión en equipos computacionales y telecomunicación, pero sin una historia muy extensa, por lo que cuando estas series no están disponibles, es necesario implementar una metodología que permita su estimación. Caso particular es el activo software, el cual no cuenta con mediciones en gran parte de la región, por lo cual se aplica una metodología conforme a los lineamentos de la OCDE y el BEA.

Para los países y los periodos de tiempo donde no existen series oficiales, los activos de equipos computacionales y telecomunicaciones son estimados a través del "commodity flow method" 8 . Este método sigue los productos desde su producción doméstica o importada a su destino final, consumo o inversión. Primero, se utilizan las encuestas industriales y estadísticas de comercio (base de datos BADECEL $^{9}$,) para obtener el gasto aparente de los equipos de oficina y computacionales y equipos de telecomunicaciones, es decir, producción nacional más importaciones menos exportaciones. Segundo, se calcula la proporción de inversión sobre el gasto aparente de dichos bienes en las matrices de insumo producto de cada país. Finalmente, para obtener las series de inversión en equipos de oficina y computacionales y equipos de telecomunicaciones esta proporción es aplicada al gasto aparente obtenido en el primer paso, como se muestra a continuación:

$$
I_{i, t}=\frac{I_{i, t}^{I O}}{\left(Q_{i, t}^{I O}+\left(M_{i, t}^{I O}-E_{i, t}^{I O}\right)\right.} *\left(Q_{i, t}+M_{i, t}-E_{i, t}\right)
$$

Donde $I_{i, j}$ es la inversión en los bienes $i$, equipos de oficina y computacionales y equipos comunicacionales, para el año $t, Q_{i t}$ es la producción doméstica, $M_{i t}$ son las importaciones y $E_{i t}$ son las exportaciones de estos bienes. El supra índice $I O$ denota el uso de las matrices de insumo producto de cada país ${ }^{10}$.

\section{B. Factor trabajo}

El cálculo de los servicios laborales permite reflejar de forma más adecuada el aporte de cada tipo de trabajador al crecimiento, al reconocer sus diferencias, como se hacen en capital, es un símil a reconocer que tienen curvas de eficiencia diferentes dependiendo de su nivel de educación.

Las horas trabajadas proveen un punto de partida para una medida económica del factor trabajo. Sin embargo, las horas pueden estar afectadas por los atributos individuales de cada ocupado, generando diferencias en la productividad de dichas horas, por lo que considerar solo una calidad constante podría ser una estimación sesgada que desconoce la heterogeneidad de la fuerza laboral.

Una mejor estimación acerca de la "calidad" del factor trabajo permitiría distinguir entre una medida que refleja la sustitución y calidad versus otra medida más simple que no logra incorporar la heterogeneidad de los trabajadores y su capital humano.

El factor trabajo es definido como el número total de horas trabajadas en un periodo dado $(\mathrm{H})$, que es igual al número total de trabajadores $(N)$ multiplicado por el número promedio de horas trabajadas por

Para más detalle véase Van Ark et al. (2002).

Base de Datos Estadísticos de Comercio Exterior de CEPAL.

Véase anexo para mayores detalles. 
trabajador $(h)$ y multiplicada por un factor de calidad $\left(L_{Q}\right)$ que incorpora las diferencias de productividad que existe entre los trabajadores.

$$
\begin{gathered}
L=N h L_{Q} \\
L_{Q}=\sum \varphi_{j} \frac{P_{j}}{P}
\end{gathered}
$$

El componente de calidad del trabajo $\left(L_{Q}\right)$, acorde a la contabilidad del crecimiento, refleja la composición laboral basada en el nivel de estudio de la población. Dicho componente es un promedio ponderado del nivel de estudio de la población $(P)$ con las tasas de retorno a la escolaridad obtenidas en ecuaciones de salarios de Mincer.

La estimación del factor trabajo de los 4 países LAKLEMS junto con incorporar el sector económico al que pertenecen suma características adicionales al nivel educacional como sexo y tramo de edad, la tasa de crecimiento del empleo $L_{\mathrm{t}}$, se expresa como una función trascendental logarítmica (translog), para los i tipos de características que se define como:

$$
\Delta \ln L_{t}=\sum \bar{v}_{t} \Delta \ln H_{i t}
$$

en donde los ponderadores $v_{t}$ están dados por el promedio de las participaciones de cada categoría en el valor de los ingresos laborales sectoriales.

Esta desagregación permite identificar la relación existente entre el aporte al crecimiento cuando se logra observar la heterogeneidad del mercado laboral. 



\section{Resultados}

Dos enfoques fueron utilizados para estimar las contribuciones a la productividad laboral. El primero de ellos, utilizado en los estudios que pueden calificarse como aquellos que utilizan el método tradicional, considera el stock del capital y el total de horas trabajadas como los factores de la ecuación (3). El segundo enfoque, explicado en detalle en las secciones anteriores, emplea las series de servicios de capital y servicios de trabajo, lo que permite capturar la heterogeneidad existente tanto en los activos del stock de capital como en las distintas características de las horas trabajadas. La serie de la productividad total de los factores (PTF), o residual, fue estimada para cada enfoque descontando del crecimiento del PIB una suma ponderada de los insumos capital y trabajo, utilizando como ponderadores la participación en los ingresos de cada insumo registrada en las cuentas nacionales ${ }^{11}$.

Comparando las estimaciones de capital, en donde la agregación de activos se realiza utilizando sus respectivos costos de uso, con el caso tradicional, donde la agregación se realiza utilizando las proporciones nominales del capital neto; se puede observar que la estimación del servicio de capital presenta una mayor contribución al crecimiento de la productividad laboral que el caso tradicional. En otras palabras, el caso tradicional subestima la aportación del capital, o de la inversión, cuando la agregación no se realiza utilizando el costo de uso como ponderador. (véase el gráfico 4). El ajuste de calidad del capital aumenta el aporte de la intensidad del capital por hora y disminuye el número de países donde es negativo (se vuelve positivo en Argentina y Brasil).

La comparación de la descomposición de los determinantes de la productividad laboral por ambos métodos, sin incluir la heterogeneidad de sus factores y luego incluyéndolas (véase el gráfico 5), muestra que el uso del método tradicional da lugar a aumentos superiores de la productividad (PTF), en contraste con la metodología ajustada que se utiliza en este estudio. Ello se explica porque aumenta el aporte, o lo que es lo mismo, el poder explicativo, de la suma de la relación capital por hora medida a costo de uso considerando las vidas medias y funciones de eficiencia especificas a cada activo, junto con el incremento de las horas trabajadas al corregirlas por educación.

\footnotetext{
11 Ante la imposibilidad de distribuir el ingreso mixto este es asignado al pago del empleo.
} 


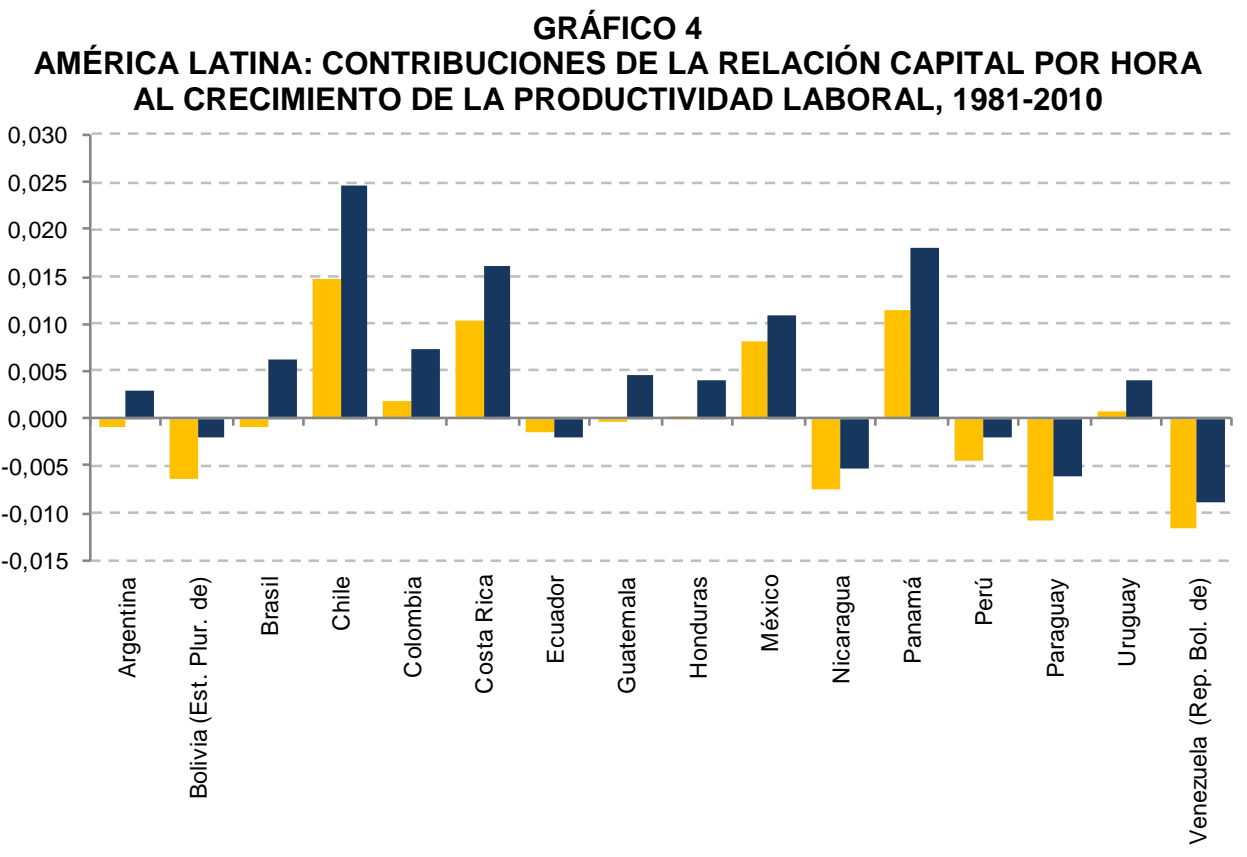

Aporte KP/HR $\quad$ Aporte SsK/Hr

Fuente: Elaboración propia.

En particular, el aporte de la calidad en las horas trabajadas muestra un aumento de estas en todos los países, tanto gracias al aumento de los años de educación como a la poca variación de las tasas de retorno. A su vez, los resultados sugieren que cuando se utiliza el valor de mercado como ponderador en la agregación de activos en el análisis de productividad, en vez del costo de uso, se subestima la aportación del capital al proceso productivo y por lo tanto, sobreestima la estimación de la productividad total de los factores. La descomposición por tipos de activos, distinguiendo entre los que tienen tasas de depreciación altas (y costes de uso altos) con los de tasas de depreciación bajas (y costes de uso iguales a productividades marginales bajas) dentro del total de capital permite medir, al menos en parte, la incorporación de progreso técnico en la inversión ${ }^{12}$, a diferencia de las metodologías que se centran en la PTF como fuente "externa" del progreso técnico.

El efecto positivo de ajuste del capital por hora es especialmente favorable en los países de mayor crecimiento (Chile, Colombia, Panamá y en menos medida Costa Rica), lo cual sugiere que no solo el monto sino también la calidad de la inversión (reflejada en este caso en el ajuste que toma en cuenta su composición y especialmente el uso de TICs) se vuelve más importante como fuente de productividad. A su vez, el análisis agregado de los 16 países permite concluir que en todos los casos hubo un aporte favorable (bastante homogéneo) de la mayor calidad de mano de obra.

Un análisis de los determinantes de la productividad por década muestra el papel que han tenido los distintos factores a través del tiempo en su aporte a la productividad laboral. De los 16 países solo

12 Parte de la compleja controversia de las escuelas de Cambridge (Reino Unido y Estados Unidos) sobre el tema de la estimación y uso del capital en un sentido agregado para la economía en su conjunto tenía que ver con el problema de no tomar en cuenta distintas tasas de depreciación para distintos tipos de equipo. Ello se estaría resolviendo con la metodología de descomponer los activos de acuerdo con sus diferentes tasas de depreciación. Aunque existían otras críticas, conviene recordar la de Joan Robinson al concepto de la productividad marginal del capital que resultaba de no tomar en cuenta sus costos diferentes de reposición: "We can compare the productivity of a given labour force equipped in one way or another (to see what differences equipment makes) but the alternative products will generally be spread over the future in different time-patterns and require different amounts of labour to produce them. If we try to express each as a perpetual even flow, by assuming that a stock of equipment once built is permanently maintained, we have to compare different replacements falling due at different dates, so that the cost of replacement is by no means a straightforward calculation. Thus we cannot compare the values of two schemes of investment in terms of their future productivity in any simple way" (Robinson 1961, p.11-2). 
dos tuvieron incrementos de ella en la década de los ochenta, y esta cifra subió a siete países en la década de los noventa y a once en la del dos mil. El aporte de la educación siempre fue positivo en todos los países y periodos, con la excepción de Venezuela (República Bolivariana de) en la década de los ochenta. El aporte de la relación capital por hora de trabajo fue principalmente negativo en los ochenta y, en los cinco países en que su aporte fue positivo, éste fue muy bajo. Esta situación cambió en los noventa, cuando fueron diez los países que registraron un aporte positivo e importante del capital por hora de trabajo, mismos que lo mantuvieron en la siguiente década.
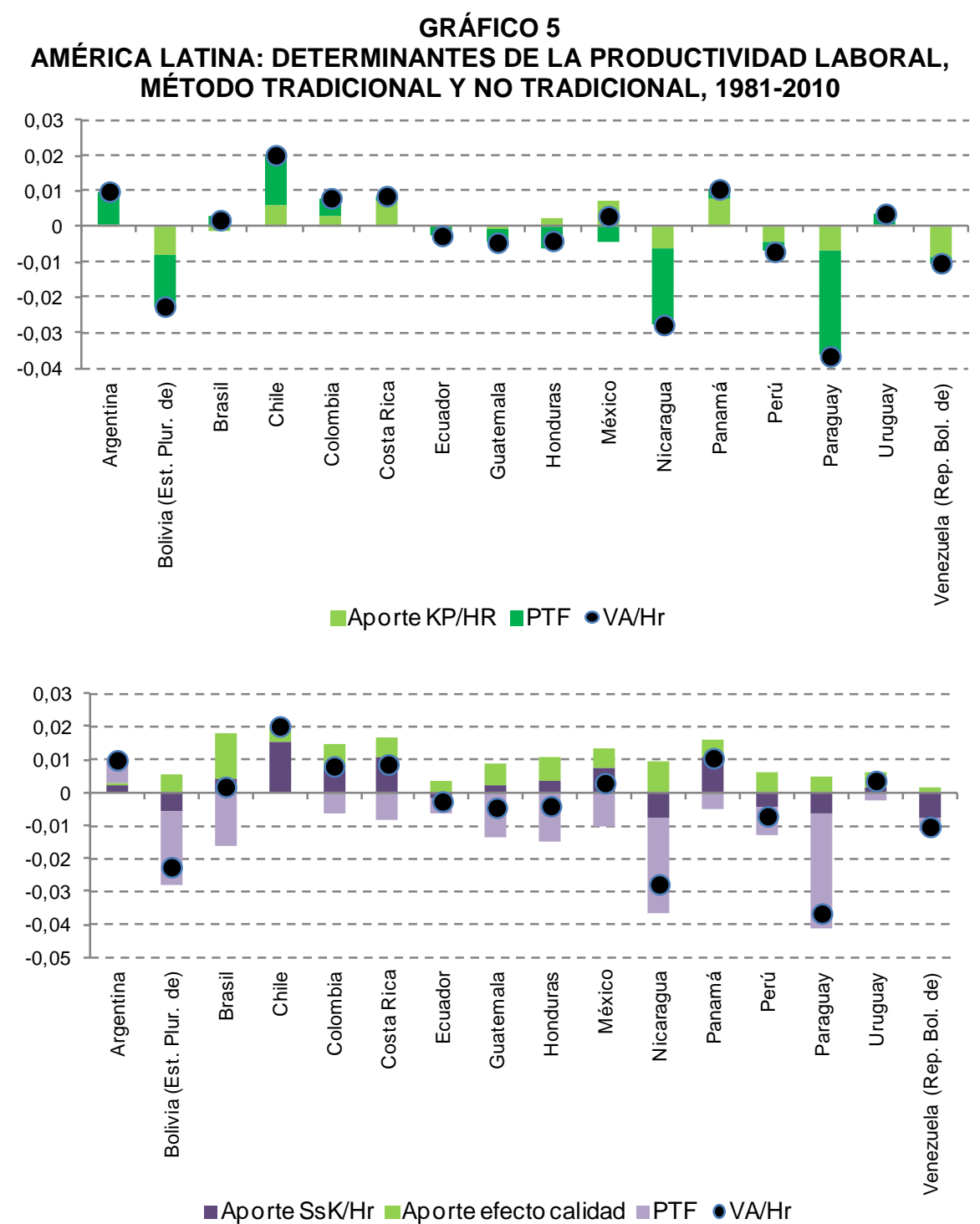

Fuente: Elaboración propia.

La estimación de la PTF sugiere que es procíclica: su aporte es positivo en el caso de los países con mayores aumentos de productividad laboral y también tiene un aporte negativo cuando hay reducciones fuertes de la productividad laboral. La contracción y "resurrección" de la PTF durante períodos de contracción y auge, documentado en estudios previos, ha conducido a la hipótesis de que estas grandes variaciones de la PTF estimada no corresponde a factores tecnológicos y que pueden resultar de restricciones financieras (Calvo, Izquierdo y Talvi, 2006). 
El hecho que la dirección (positiva o negativa) de estas variaciones coinciden con la dirección del aporte de la inversión (ajustada), sugiere que la PTF está reflejando el grado de utilización de la capacidad instalada: en momentos de contracción cae —como lo hace la inversión- y en momentos de auge se eleva, también junto con la inversión (véase el grafico 6). A su vez, y congruente con los mayores aportes de la inversión y del trabajo (ajustados), el tamaño del aporte de la PTF, positivo o negativo, se reduce con el avance de las décadas (véase gráfico 6), todo lo cual refuerza su caracterización no solo como un factor residual que refleja la existencia de variables no recogidas en el modelo (el grado de utilización de la capacidad instalada, entre otras), sino también su capacidad débil o inexistente para medir el progreso técnico.

\section{GRÁFICO 6 AMÉRICA LATINA: DETERMINANTES DE LA PRODUCTIVIDAD LABORAL, 1980-2010}
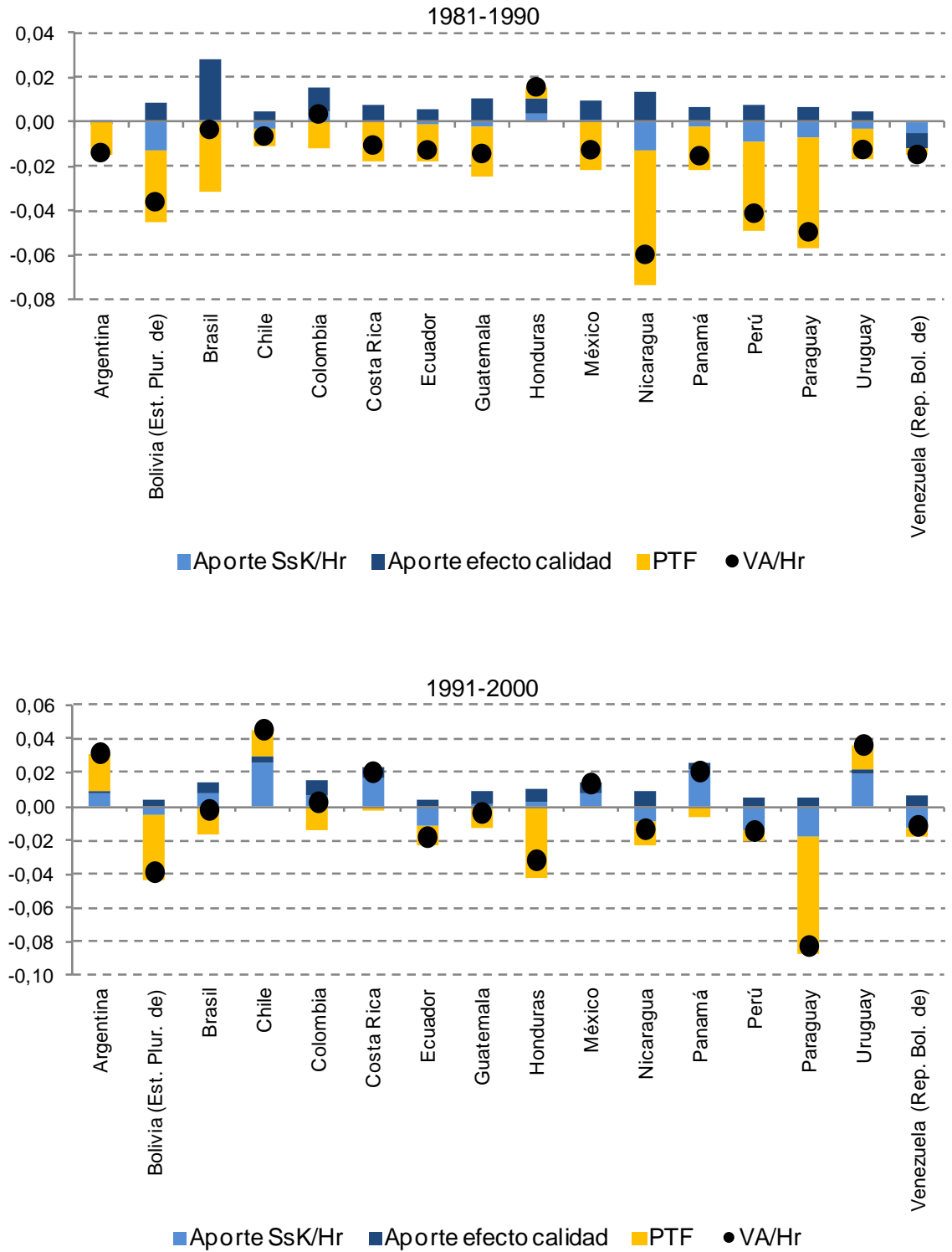
Gráfico 6 (conclusión)

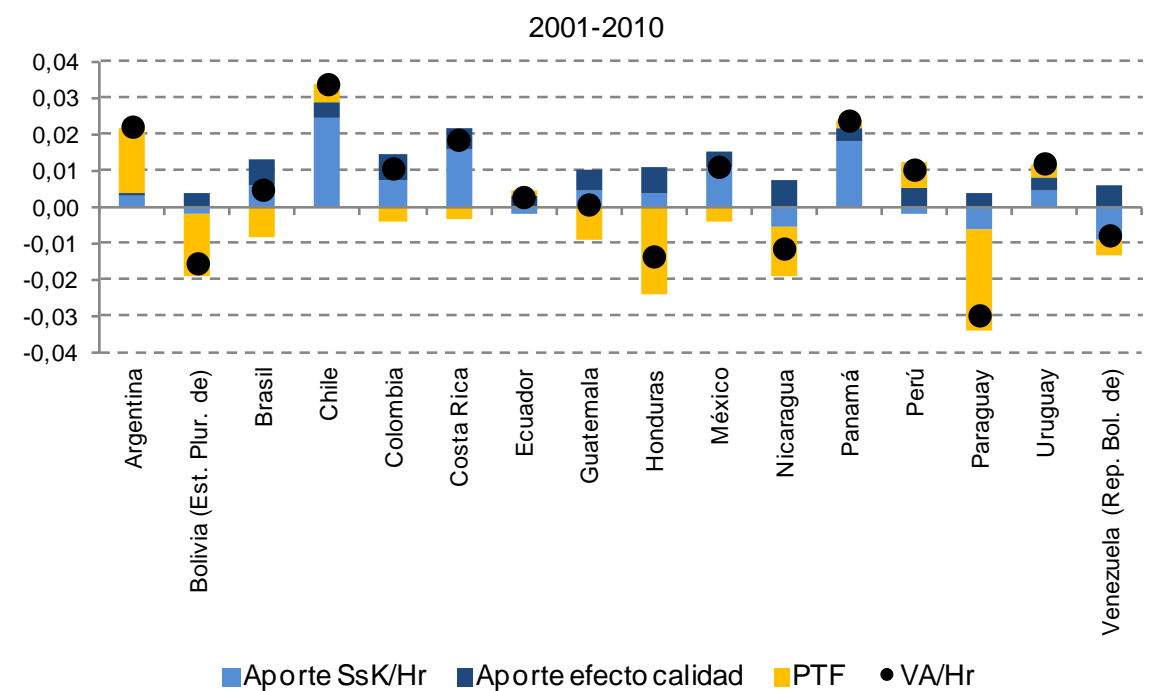

Fuente: Elaboración propia.

\section{GRÁFICO 7 \\ ARGENTINA, BRASIL, CHILE Y MÉXICO: DETERMINANTES DE LA PRODUCTIVIDAD \\ LABORAL, 1994-2008}

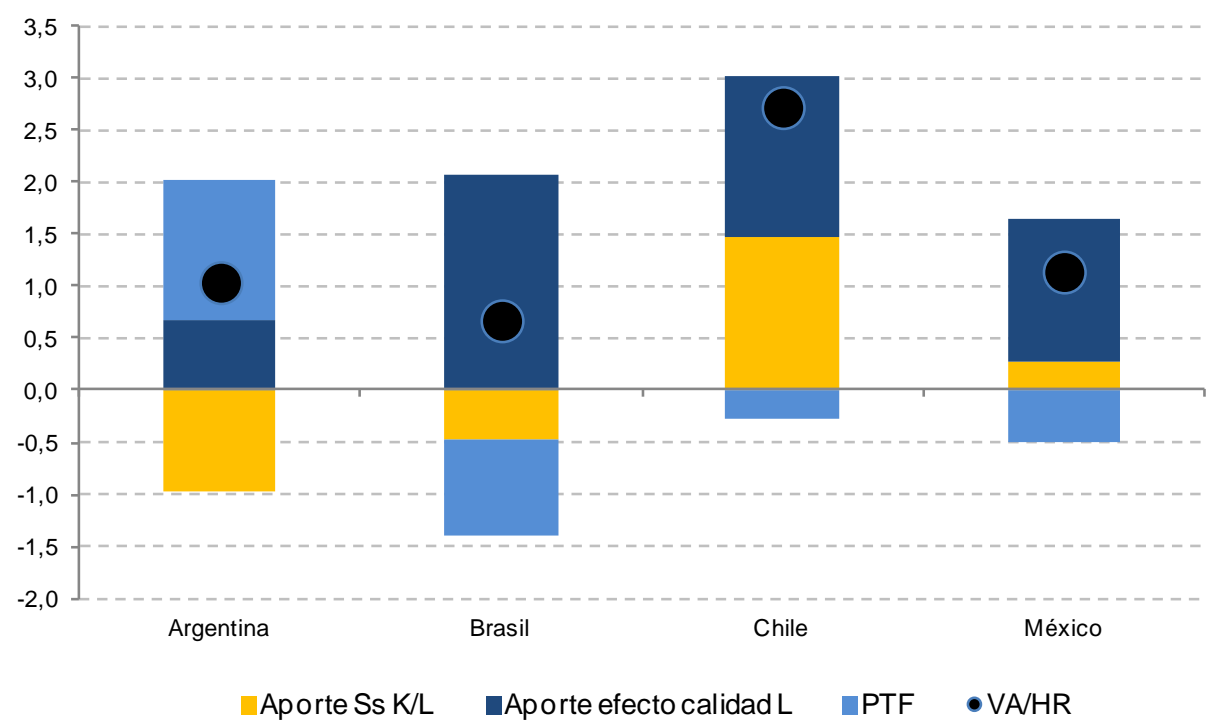

Fuente: Elaboración propia, base de datos LAKLEMS.

Nota: Datos para Brasil van desde 1996 a 2008.

El análisis de los aportes a la evolución global de la productividad laboral de los cuatro países (Argentina, Brasil, Chile y México) para los cuales hay datos sectoriales disponibles como resultado del proyecto KLEMS-LA, confirma el aporte positivo de la mejora en la calidad de mano de obra en todos los casos (véase el gráfico 7). En el caso del Brasil el aumento de la calidad del trabajo es el único aporte positivo al crecimiento de la productividad laboral globalmente, que podría explicarse como resultado de un aumento importante de empleo, cada vez más calificado — aunque partiendo de una base limitada_- 
en ese país. Este mayor crecimiento del número de horas trabajadas en comparación con el capital explicaría el aporte global negativo del capital/hora trabajada a la productividad laboral en Brasil.

Chile y México se caracterizan por presentar lo que podría calificarse como un patrón "normal" de aumento de la productividad laboral: refleja aportes positivos originados tanto por un incremento de la inversión por hora trabajada como por mejoras de la calidad de la mano de obra, aunque el aporte de la inversión es mayor en Chile y explica su mayor productividad laboral en comparación con México.

Mientras que Brasil, Chile y México presentan una estimación del aporte de la PTF a la productividad laboral que es negativa, en el caso de Argentina es positiva, además del aporte de la calidad de mano de obra (véase el gráfico 7), similar a lo encontrado por Jorgenson y Vu (2010). Al evaluarse la evolución de la productividad para este conjunto de países (véase el gráfico 8) tiende a confirmarse la correspondencia entre auge y crisis y aumento y disminución de la PTF (Calvo, Izquierdo y Talvi, 2006), que en el caso de la Argentina sugiere que ante la insuficiente inversión ha habido momentos (1994-1996 o 2005-2007) que se podrían calificar como mayor utilización de la capacidad instalada. También hubo ajustes rápidos en el empleo de mano de obra calificada (en 2004) después de una inicial ampliación del empleo menos calificado (2003) al superarse la crisis de 2001-2002.

\section{GRÁFICO 8 \\ ARGENTINA, BRASIL, CHILE Y MÉXICO: DETERMINANTES DE LA PRODUCTIVIDAD LABORAL POR AÑO, 1994-2008}
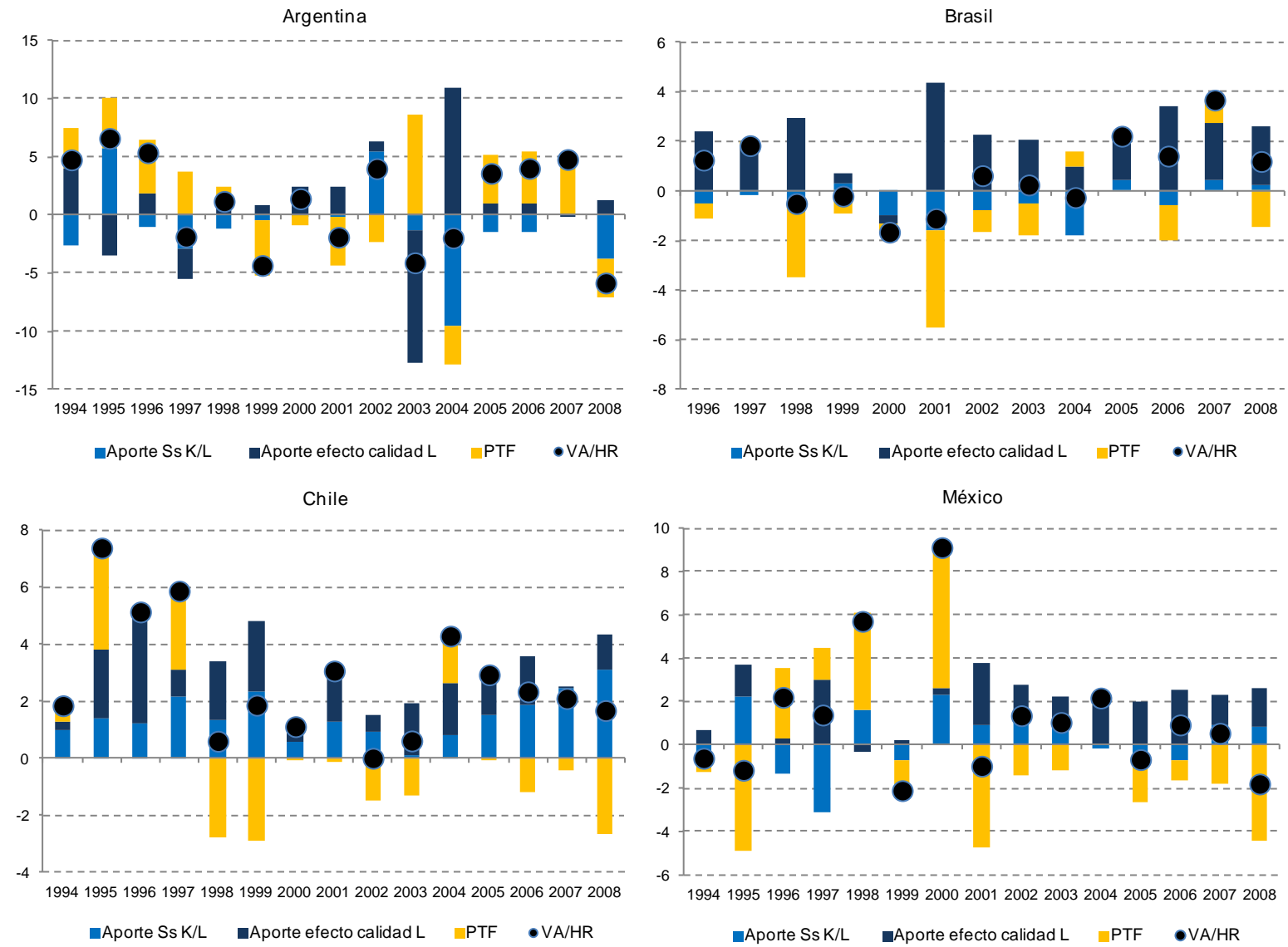

Fuente: Elaboración propia, base de datos LAKLEMS.

Nota: Datos para Brasil van desde 1996 a 2008.

La evolución de los aportes a la productividad en Brasil, a su vez, confirma el aporte importante de las mejoras de la calidad de la mano de obra a lo largo del tiempo, y sugiere que la inversión por hora 
trabajada (ajustada) ha sido insuficiente para aumentar la productividad de manera más categórica. El aporte de la inversión por hora trabajada ha sido mayor en México que en el caso de Argentina o Brasil, pero el contraste con Chile — donde el aporte de la inversión por hora trabajada no solo es mayor sino positivo todos los años - también sugiere que en México — como en Argentina y Brasil— ha sido insuficiente, tanto en términos de monto como de calidad (véase el gráfico 4).

$\mathrm{Al}$ analizar los aportes a la productividad laboral por sector (véase el gráfico 9), se destacan algunos rasgos comunes. Primero, para los países para los cuales existe información disponible, la agricultura tiende a ser uno de los sectores donde más aumenta la productividad laboral. En el caso de Brasil y Chile ello está asociado con un aumento importante del aporte estimado de la PTF, que en los hechos probablemente refleje una disminución del empleo de la mano de obra no calificada en el sector. En México el aumento de la productividad laboral en el sector agrícola está asociado principalmente con un aumento de la inversión por hora trabajada.

Segundo, en México y Brasil las variaciones de la productividad laboral varían significativamente por sectores, mientras que en Chile y Argentina predominan los aumentos de la productividad en la mayoría de sectores. En el caso de Chile estos aumentos coinciden con un aumento del aporte positivo de la inversión, mientras que en Argentina la mayor productividad coincide con un aporte positivo importante de la PTF, lo cual posiblemente refleje menores niveles de inversión que resultan en una mayor utilización de la capacidad instalada en cada sector, como ya se indicó. En México la productividad aumenta en los sectores transables y disminuye en los no transables, y Brasil manifiesta la mayor heterogeneidad: mientras la productividad laboral experimenta un fuerte aumento en agricultura se reduce en la industria, la construcción y en varios de los sectores de servicios.

Tercero, los aumentos tienden a ser mayores en los sectores transables (representados por la agricultura, la minería y la industria), en contraste con los no transables (el resto, consistente en construcción y servicios) $)^{13}$, aunque con variaciones entre países. La productividad del sector industrial aumenta en mayor medida en Chile y más modestamente en Argentina y México, mientras que cae en Brasil.

Cuarto, la estimación del aporte de la PTF tiende a ser negativa en todos los sectores, con la excepción de Argentina, donde es positiva para casi todos los sectores ${ }^{14}$. En general, el patrón sectorial del aporte de la PTF coincide con el patrón que caracteriza el conjunto de países de la región, con aportes mayores en aquellos sectores con los aumentos o disminuciones más fuertes de la productividad laboral. Un caso que ilustra el aporte negativo de la PTF como reflejo de capacidad no utilizada es la electricidad, gas y agua de Chile, donde el cambio de la matriz energética resultó en un aumento significativo de la inversión que no se utilizó (por la interrupción del envío de gas desde Argentina) o cuya eficiencia es limitada (poco valor agregado generado por equipo que utiliza hidrocarburos importados a altos costos).

Quinto, en los cuatro países el aporte de la calidad de la mano de obra es positivo, con mayores variaciones del aporte positivo en el caso de México en comparación con los demás países.

13 En la práctica existen servicios que son transables, pero se establece esta separación como una aproximación analítica.

14 Cobertura de las encuestas de empleo no permiten capturar la composición del empleo para minería y agricultura. 


\section{GRÁFICO 9 \\ ARGENTINA, BRASIL, CHILE Y MÉXICO: DETERMINANTES DE LA PRODUCTIVIDAD LABORAL POR SECTOR, 1994-2008}
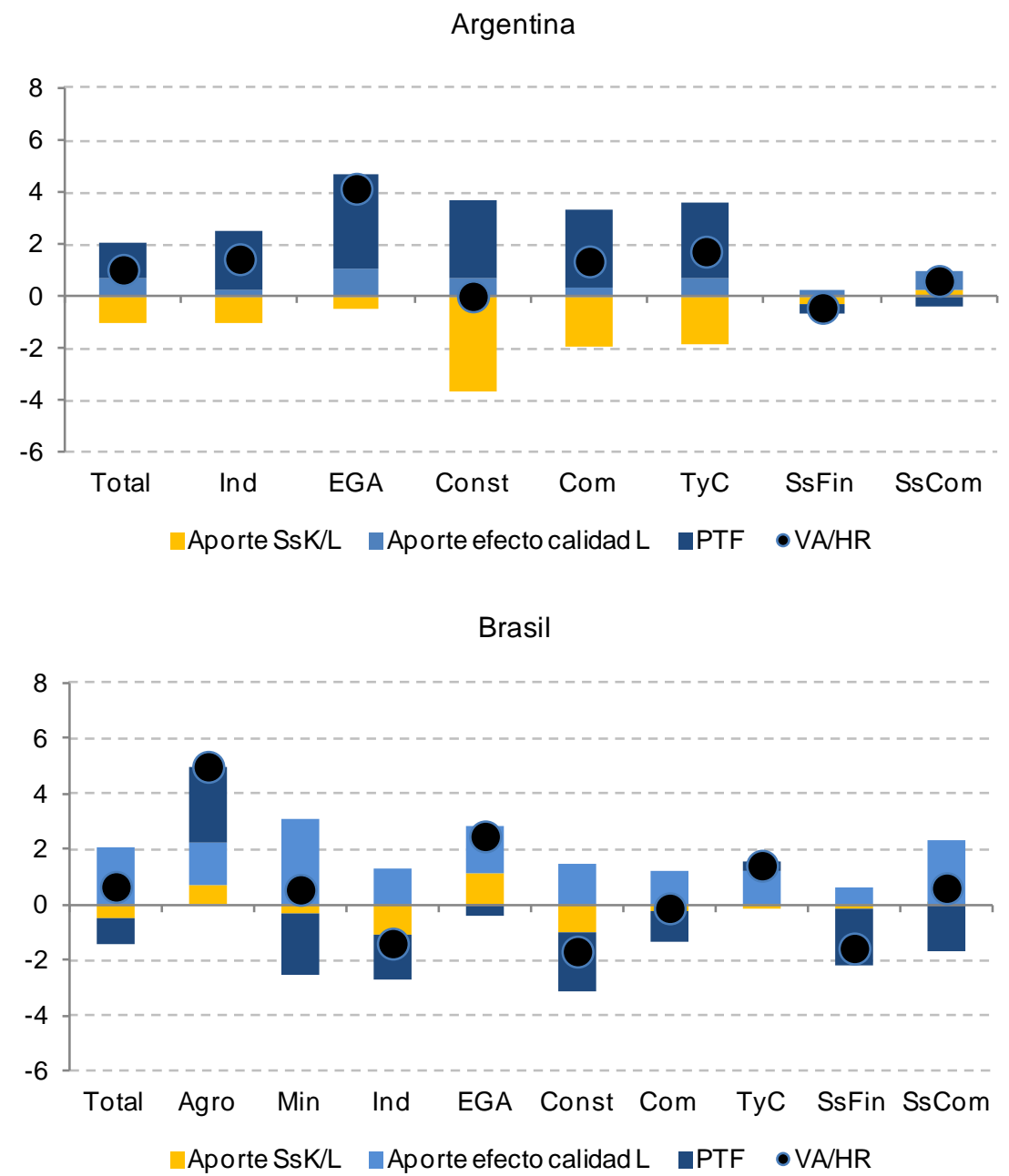

Chile

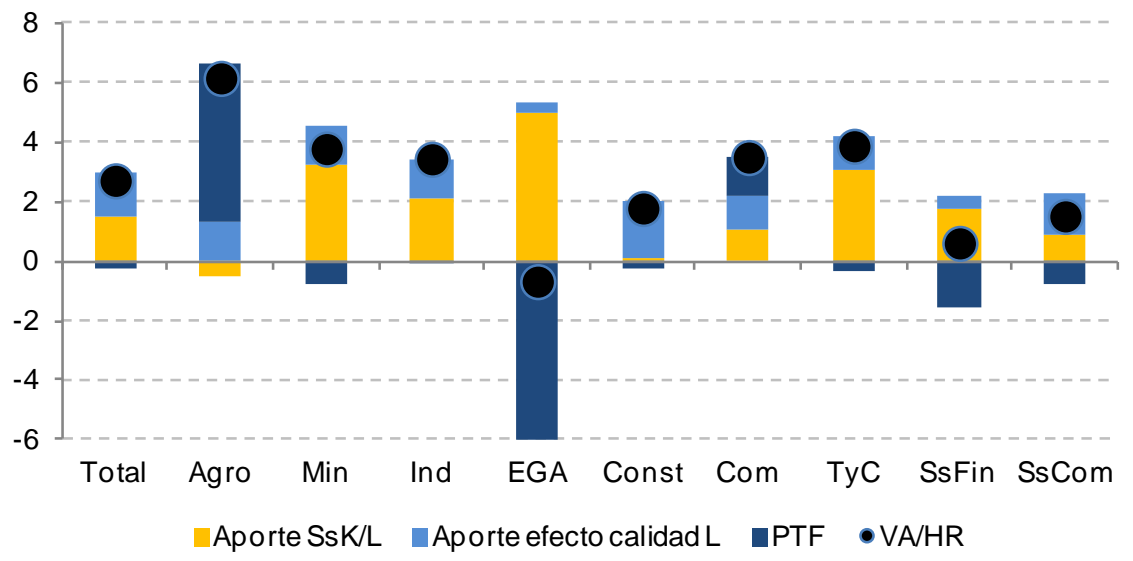


Gráfico 9 (conclusión)

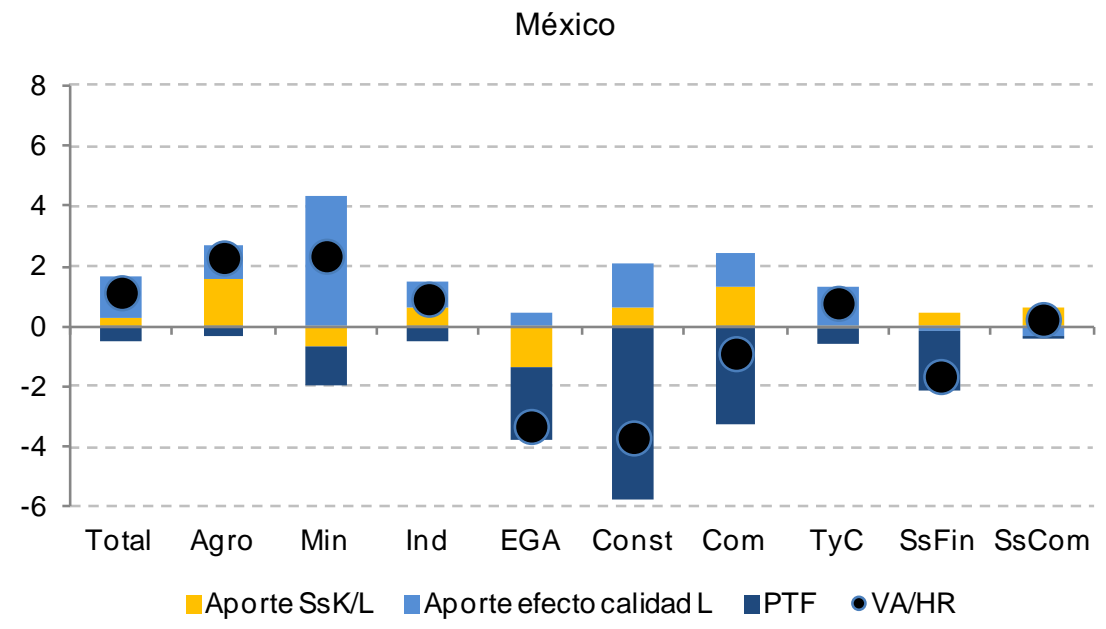

Fuente: Elaboración propia, base de datos LAKLEMS.

Nota: Datos para Brasil van desde 1996 a 2008. 



\section{Conclusiones}

La baja productividad laboral es la principal causante del pobre crecimiento económico que han tenido los países en América Latina, durante las últimas tres décadas. Su aporte promedio al crecimiento fue negativo $(-0,3 \%)$ y siendo el aumento de las horas trabajas el sustento en el crecimiento de la región.

Además, a lo largo del periodo 1980-2010, la tasa de crecimiento de la productividad laboral en los países de América Latina fue inferior a la de EE.UU., provocando un aumento de la brecha con respecto a Estados Unidos de 12 puntos porcentuales, siendo Chile el único país que la disminuyó. Estos resultados de baja productividad son la principal motivación para este estudio, que busca dar respuesta a cuáles son sus principales determinantes y la forma correcta de medirlos.

De manera similar a como los trabajadores son repositorios del stock de capital humano y prestan servicios medidos en horas trabajadas, los bienes de capital representan un stock que provee un flujo de servicios, que son insumos en el proceso productivo. La correcta estimación de su aporte requiere capturar la heterogeneidad de sus activos. Sin embargo, mientras que los trabajadores reciben una remuneración a cambio de los servicios prestados, en general, los bienes de capital son propiedad de la empresa que los utiliza, por lo que no existe un registro de la remuneración por los servicios prestados por los bienes de capital.

En consecuencia, la medición del aporte del insumo capital al proceso productivo plantea la necesidad de hacer una correcta estimación de los ponderadores utilizados en la agregación de los distintos tipos de bienes de capital, lo cual debe realizarse utilizando el costo de uso de cada uno de ellos y no sus precios de mercado como tradicionalmente se hace.

El cálculo tradicional de la medición del capital para los países de América Latina sugiere que ha predominado el aporte de la PTF a la productividad laboral, y que el aporte de la intensidad de la inversión por hora ha sido positivo en algunos casos y negativo en otros, con predominio de este último efecto. Sin embargo, al incluir en la metodología de medición a la heterogeneidad de los activos de capital, y especialmente el aporte de las TICs — que puede tomarse como un indicador de la calidad de la inversión - el aporte de la inversión por hora trabajada a la productividad laboral aumenta, y disminuye el número de países donde es negativa, poniendo de manifiesto que con la metodología tradicional se subestima el aporte real de la inversión (con progreso técnico incorporado) a la productividad laboral. 
El análisis más detallado de Argentina, Brasil, Chile y México refuerza lo anterior. Chile y México comparten lo que podría calificarse como un patrón "normal" de aumento de productividad laboral, dado por aportes positivos de una mayor inversión por hora trabajada y de mejoras de la calidad de mano de obra. En Argentina, el aumento de la productividad está asociado con aportes de la PTF y de calidad de mano de obra, y en Brasil, la fuente casi exclusiva de aumentos de la productividad laboral resulta ser las mejoras en la calidad de la mano de obra.

El aporte de la inversión por hora trabajada ha sido mayor en México que en el caso de Argentina o Brasil, pero el contraste con Chile - donde el aporte de la inversión por hora trabajada no solo es mayor sino positivo todos los años - también sugiere que en México — junto con Argentina y Brasilha sido insuficiente, tanto en términos de monto como de calidad. El hecho que del conjunto de países analizados aquellos con mayor crecimiento de su productividad laboral también son aquellos con un mayor aporte de la inversión por hora trabajada confirma la necesidad de aumentar la inversión, tanto en cantidad como en calidad.

Por otra parte, el hecho que la dirección (positiva o negativa) de las variaciones del aporte de la PTF coinciden con la dirección del aporte de la inversión (ajustada), sugiere que la PTF está reflejando el grado de utilización de la capacidad instalada: en momentos de contracción cae — como lo hace la inversión- $-\mathrm{y}$ en momentos de auge se eleva, también junto con la inversión. Esto ocurre tanto al tomar a los países como a los sectores como objeto del estudio de la productividad laboral. A su vez, y congruente con los mayores aportes de la inversión y del trabajo (ajustados), el tamaño del aporte de la PTF estimada a la productividad laboral, positivo o negativo, se reduce con el avance de las décadas. Todo ello refuerza su caracterización no solo como un factor residual que refleja la existencia de variables no recogidas en el modelo (el grado de utilización de la capacidad instalada), sino también su capacidad débil o inexistente para medir el progreso técnico.

En cuanto al aporte del trabajo a la productividad laboral, existe homogeneidad en todos los países de la región respecto al aporte de la educación en la mejora de su fuerza de trabajo, lo cual se refleja en un aporte positivo y generalizado de la calidad del trabajo al crecimiento de la productividad laboral. Ello también confirma la importancia de la formación y capacitación de la mano de obra como requisito para aumentar la productividad.

El análisis sectorial permite confirmar las apreciaciones anteriores y obtener conclusiones adicionales. Entre éstas últimas están el aumento mayor de la productividad laboral experimentado por el sector agrícola, un aumento más homogéneo de la productividad laboral en Argentina y Chile en contraste con la heterogeneidad sectorial de Brasil y México, y una tendencia a que los aumentos de productividad hayan sido mayores en los sectores transables que en los no transables. 


\section{Bibliografía}

Abramovitz, M. (1993), “The Search for the Sources of Growth: Areas of Ignorance, Old and New”, The Journal of Economic History, vol. 53, Issue 2, junio: 213-243.

Aravena, C., Jofré, J., and Villarreal, F. (2009), Estimación de Servicios de Capital y Productividad para América Latina. Serie Estudios Estadisticos y Prospectivos, CEPAL.

Calvo, G.A., A. Izquierdo and E. Talvi (2006), "Phoenix Miracles in Emerging Markets: Recovering without Credit from Systemic Financial Crisis”, NBER Working Paper 1201, Cambridge, MA, marzo.

Fraumeni, B. (1997), The Measurement of Depreciation in the U.S. National Income and Product Accounts. Survey of Current Business, pags. 7-23.

Griliches, Z. (1994), Productivity, R\&D, and the Data Constraint. American Economic Review, 84(1):1-23.

Harberger, A. (1978), Perspectives on Capital and Technology in Less Developed Countries. In Artis, M. and Nobay, A., editors, Contemporary Economic Analysis. Croom Helm, Londres.

Harchaoui, T. M. and Tarkhani, F. (2002), A Comprehensive Revision of Statistic's Canada Estimates of Capital Input for Productivity Accounts. Technical report, Statistics Canada, www.statcan.gc.ca

Hofman A. y C. Aravena (2013), Productivity and Economic Growth in Latin America - LA KLEMS productivity data base (mimeo).

Hulten, C. R. and Wykoff, F. C. (1981a), Economic Depreciation and the Taxation of Structures in United States Manufacturing Industries: An Empirical Analysis. In Usher, D., editor, The Measurement of Capital, pages 83-120. University of Chicago Press, Chicago.

(1981b), The Estimation of Economic Depreciation using Vintage Assets Prices: An Aplication of the Box-Cox Power Transformation. Journal of Econometrics, 15:367-396. (1981c), The Measurement of Economic Depreciation. In Hulten, C. R., editor, Depreciation, Inflation and the Taxation of Income from Capital, pages 81-125. The Urban Institute Press, Washington, D.C.

ILO (2009), Estimates and projections of the economally active population:1980-2020 (fifth edition). Methodological description

Jorgenson, D. W. and Griliches, Z. (1967), The Explanation of Productivity Change. Review of Economic Studies, (34):249-283.

Jorgenson, Dale W., Frank M. Gollop and Barbara M. Fraumeni (1987), Productivity and U.S. Economic Growth. Harvard University Press, Cambridge, MA.

Jorgenson, D. W., Ho, M. S. and Stiroh, K. J. (2005), Information Technology and the American Growth Resurgence, MIT Press, Cambridge, MA. 
Jorgenson, D. W. and Khuong Minh Vu (2010), "Latin America and the world economy” in Innovation and economic development: The impact of information and communication technologies in Latin America, Cimoli, Hofman and Mulder, (eds.): Cheltenham [u.a.]: Elgar, ISBN 978-1-8498-0241-3, 2010, págs. 19-42.

Mas, M., Pérez, F., and Uriel, E. (2005), El stock y los servicios de capital en España, nueva metodología. Fundación BBVA, Bilbao.

OCDE (1991), Flows and Stocks of Fixed Capital (1969-89). OECD Department of Economics and Statistics. (2001a), Measuring Capital. Measurement of Capital Stocks, Consumption of Fixed Capital and Capital Services. Organización para la Cooperación y Desarrollo Económicos, París. (2001b), Measuring Productivity. Measurement of Aggregate and Industry-Level Productivity Growth. Organización para la Cooperación y Desarrollo Económicos, París.

Robinson, J. (1961), Exercises in Economic Analysis, London, Macmillan \& Co. Ltd.

Vries de, G., Mulder, N., Borgo dal, M., and Hofman, A. (2007), ICT Investment in Latin America: Does it Matter for Economic Growth? Universidad de Groningen. 


\section{Anexo}




\section{Datos}

\section{Capital}

Las series de formación bruta de capital fijo (FBCF) debe estar desagregada por sector económico y tipos de activos, detalle en cuadro A.1 y cuadro A.2. La desagregación no coincide necesariamente con las clasificaciones de la FBCF publicadas por los institutos nacionales de estadísticas y los Bancos Centrales de los países de estudio. Estas diferencias se originan en la falta de desagregación en algunos activos y en modificaciones metodológicas introducidas en el periodo de análisis por cambios en los años de referencia de las cuentas nacionales. Por ello, la tarea abordada no sólo supone el enlace de magnitudes sino que también, en la medida de lo posible, incorpora la necesidad de realizar una homogeneización previa de dichas magnitudes.

\begin{tabular}{l} 
CUADRO A.1 \\
$\qquad$ SECTORES ECONÓMICOS \\
\hline Agricultura, caza y pesca \\
Minas y canteras \\
Industria manufacturera \\
Electricidad, gas y agua \\
Construcción \\
Comercio, hoteles y restaurantes \\
Transporte y comunicaciones \\
Servicios financieros \\
Servicios comunales y sociales \\
\hline Fuente: Elaboración propia. \\
Cotros \\
Productos TIC \\
Maquinaria de oficina y equipo informático \\
Equipos de telecomunicaciones \\
Software \\
Construcción
\end{tabular}

Fuente: Elaboración propia.

Con base en las cifras oficiales de cada país y la estimación de la totalidad o parte de los activos TIC se efectuó un ejercicio de aproximación a la descomposición planteada, para más detalle Hofman y Aravena (2013). 


\section{Empleo}

Los datos acerca de horas e ingreso comprenden el período de 1990 a 2009, información extraída de las encuestas de hogares de cada país. En el caso de Argentina, se utilizó la Encuesta Permanente de Hogares juntos con las cuentas nacionales. Para el caso de Brasil, se recurrió a la Encuesta Nacional para Muestra de Hogares (PNAD) y el Informe Anual de Informaciones Sociales (RAIS). En Chile, la información se obtuvo a través de la Encuesta Nacional de Empleo (ENE) y la Encuesta Suplementaria de Ingresos (ESI), y para México los datos fueron recopilados de la Encuesta Nacional de Ocupación y Empleo (ENOE), la Encuesta Nacional de Empleo (ENE) y los Censos Económicos (1993 y 1998).

Los micro datos se trabajaron y enlazaron con el objeto de construir estimaciones homogéneas metodológicamente entre los cuatro países para los ocupados según nivel de estudios, edad, género y sector de actividad donde se desempeñan, lo cual es imprescindible para aplicar la metodología KLEMS. Con esta información fue posible la estimación de los ocupados en términos de horas efectivamente trabajadas y los ingresos laborales, asociados a cada una de sus características, cuadro A.3.

\section{CARACTERÍSTICAS DE CLASIFICACIÓN}

\begin{tabular}{ll}
\hline & Agricultura, caza y pesca \\
& Minas y canteras \\
& Industria manufacturera \\
& Electricidad, gas y agua \\
Sectores económicos & Construcción \\
& Comercio, hoteles y restaurantes \\
& Transporte y comunicaciones \\
& Servicios financieros \\
& Servicios comunales y sociales \\
\hline Sexo & Femenino \\
& Masculino \\
\hline Tramo de edad & $15-29$ años \\
& $30-49$ años \\
& 50 y más años \\
\hline Calificación baja \\
Calificación media \\
Calificación alta
\end{tabular}

Fuente: Elaboración propia. 


\section{Serie}

\section{E P A L Macroeconomía del Desarrollo}

\section{Números publicados}

\section{Un listado completo así como los archivos pdf están disponibles en www.cepal.org/publicaciones}

140. El desempeño mediocre de la productividad laboral en América Latina: una interpretación neoclásica, Claudio Aravena y Juan Alberto Fuentes (LC/L.3725), 2013.

139. Generating inclusive and sustainable growth. The role of policy and multilevel fiscal institutions, Ehtisham Ahmad (LC/L.3718), 2013.

138. Políticas fiscales para el crecimiento y la igualdad, Ricardo Martner, Andrea Podestá e Ivonne González (LC/L.3716), 2013.

137. Desarrollo minero y conflictos socioambientales. Los casos de Colombia, México y Perú, Miryam Saade (LC/L.3706), 2013.

136. Rasgos estilizados de la relación entre inversión y crecimiento en América Latina y el Caribe, 1980-2012, Luis Felipe Jiménez y Sandra Manuelito (LC/L.3704), 2013.

135. Impactos de las reformas recientes de política fiscal sobre la distribución de los ingresos. El caso de Perú, Andrés Escalante (LC/L.3699), 2013.

134. Política fiscal y crecimiento económico. Consideraciones microeconómicas y relaciones macroeconómicas, José Félix Sanz-Sanz e Ismael Sanz Labrador (LC/L.3638), 2013.

133. Política tributaria en América Latina: agenda para una segunda generación de reformas, Juan Carlos Gómez Sabaíni y Dalmiro Morán (LC/L.3632), 2013.

132. Descentralización, inversión pública y consolidación fiscal: hacia una nueva geometría del triángulo, Roberto Fernández Llera (LC/L.3622), 2013.

131. La arquitectura financiera mundial y regional a la luz de la crisis, José Antonio Ocampo (LC/L.3584), 2013.

130. Política tributaria para mejorar la inversión en América Latina, Claudio Agostini y Michel Jorratt (LC/L.3589), 2013.

129. La inversión y el ahorro en América Latina: nuevos rasgos estilizados, requerimientos para el crecimiento y elementos de una estrategia para fortalecer su financiamiento, Sandra Manuelito y Luis Felipe Jiménez, (LC/L.3603), 2013.

128. Interacciones intergubernamentales entre los impuestos sobre el petróleo y el gas y la protección ambiental, Giorgio Brosio (LC/L.3583), 2013.

127. Política fiscal e inversión: Un enfoque sistémico y de crecimiento inclusivo, José María Fanelli, (LC/L.3556), 2013.

126. Elites económicas, desigualdad y tributación, Juan Pablo Jiménez y Andrés Solimano, (LC/L.3552), 2012.

125. La reforma tributaria uruguaya de 2006: algunas consideraciones de economía política y comportamental, Andrés Rius (LC/L.3550), 2012.

124. Informalidad y tributación en América Latina: Explorando los nexos para mejorar la equidad, Juan Carlos Gómez Sabaíni y Dalmiro Morán, (LC/L.3534), 2012.

123. Clase media y política fiscal en América Latina, Diego Avanzini, (LC/L.3527), 2012.

122. Crecimiento, empleo y distribución de ingresos en América Latina, Jürgen Weller (LC/L.3516), 2012.

121. Revenue sharing: the case of Brazil’s ICMS, José Manuel Arroyo, Juan Pablo Jiménez and Carlos Mussi (LC/L.3489), 2012.

120. Estimación de la recaudación potencial del impuesto a la renta en América Latina, Darío Rossignolo (LC/L.3484), 2012.

119. Imposición a la renta personal y equidad en América Latina: Nuevos desafíos, J. C. Gómez Sabaíni, J. Pablo Jiménez y Darío Rossignolo (LC/L.3477), 2012.

118. Tax structure and tax evasion in Latin America, Juan Carlos Gómez Sabaíni and Juan Pablo Jiménez (LC/L.3455), 2012.

117. Commodities, choques externos e crescimento: reflexões sobre a América Latina, Ricardo de Medeiros Carneiro (LC/L.3435), 2012.

116. Fragilidad externa o desindustrialización: ¿Cuál es la principal amenaza de América Latina en la próxima década?, Roberto Frenkel y Martín Rapetti (LC/L.3423), 2011.

115. Desafíos e implicancias fiscales de la inversión en infraestructura baja en carbono, Martina Chidiak y Verónica Gutman (LC/L.3403), 2011. 